\title{
A Joint Transmission Power Control and Duty- Cycle Approach for Smart Healthcare System
}

Ali Hassan Sodhro, Sandeep Pirbhulal, Gul Hassan Sodhro, Andrei Gurtov, Muhammad Muzamal and Zongwei Luo

The self-archived postprint version of this journal article is available at Linköping University Institutional Repository (DiVA):

http://urn.kb.se/resolve?urn=urn:nbn:se:liu:diva-153119

N.B.: When citing this work, cite the original publication.

Sodhro, A. H., Pirbhulal, S., Sodhro, G. H., Gurtov, A., Muzamal, M., Luo, Z., (2018), A Joint Transmission Power Control and Duty-Cycle Approach for Smart Healthcare System, IEEE Sensors Journal. https://doi.org/10.1109/JSEN.2018.2881611

Original publication available at:

https://doi.org/10.1109/JSEN.2018.2881611

Copyright: Institute of Electrical and Electronics Engineers (IEEE)

http://www.ieee.org/index.html

(C)2018 IEEE. Personal use of this material is permitted. However, permission to reprint/republish this material for advertising or promotional purposes or for creating new collective works for resale or redistribution to servers or lists, or to reuse any copyrighted component of this work in other works must be obtained from the IEEE. 


\title{
A Joint Transmission Power Control and Duty-Cycle Approach for Smart Healthcare System
}

\author{
Ali Hassan Sodhro", Sandeep Pirbhulal", Gul Hassan Sodhro, Andrei Gurtov, Muhammad Muzammal, Zongwei Luo*
}

\begin{abstract}
Emerging revolution in the healthcare has caught the attention of both the industry and academia due to the rapid proliferation in the wearable devices and innovative techniques. In the mean-time, Body Sensor Networks (BSNs) have become the potential candidate in transforming the entire landscape of the medical world. However, large battery lifetime and less power drain are very vital for these resource-constrained sensor devices while collecting the bio-signals. Hence, minimizing their charge and energy depletions are still very challenging tasks. It is examined through large real-time data sets that due to the dynamic nature of the wireless channel, the traditional predictive transmission power control (PTPC) and a constant transmission power techniques are no more supportive and potential candidates for BSNs. Thus this paper first, proposes a novel joint transmission power control (TPC) and duty-cycle adaptation based framework for pervasive healthcare. Second, adaptive energy-efficient transmission power control (AETPC) algorithm is developed by adapting the temporal variation in the on-body wireless channel amid static (i.e., standing and walking at a constant speed) and dynamic (i.e., running) body postures. Third, a Feedback Control-based duty-cycle algorithm is proposed for adjusting the execution period of tasks (i.e., sensing and transmission). Fourth, system-level battery and energy harvesting models are proposed for body sensor nodes by examining the energy depletion of sensing and transmission tasks. It is validated through Monte Carlo experimental analysis that proposed algorithm saves more energy of $11.5 \%$ with reasonable packet loss ratio (PLR) by adjusting both transmission power and duty-cycle unlike the conventional constant TPC and PTPC methods.
\end{abstract}

Index Terms - Duty-cycle, Body Posture, Smart Healthcare, energy harvesting, AETPC, BSN, PTPC, Constant TPC

\section{INTRODUCTION}

$\mathrm{P}$ ervasive and economical healthcare is the dire need of the aging society, due to the emerging technological trends of-

Ali Hassan Sodhro is with the IDA-Computer and Information Science Department Linkoping University Sweden and Electrical Engineering Department Sukkur IBA, University, Sukkur, Sindh, Pakistan. (Email: ali.hassan@iba suk.edu.pk, aliso78@liu.se)

Sandeep Pirbhulal is with CAS, SIAT, Shenzhen, China (Email:sandeep@siat.ac.cn)

Gul Hassan Sodhro is with Department of Physics Shah Abdul Latif, University, Khairpur Mirs, Khairpur, Sindh, Pakistan (Email: hassangul1183@gmail.com).

Andrei Gurtov, is with the IDA-Computer and Information Science Department of Linkoping University, Linkoping, Sweden (Email: Andrei.gurtov@liu.se)

Muhammad Muzamal, Department of Computer Science Bahria University, Islamabad, Pakistan (Email: muzammal@ bui.edu.pk)

*Zongwei Luo is with Shenzhen Key Laboratory of Computational Intelligence, computer science and Engineering department of Southern University of Science and Technology, Shenzhen, China (Email:luozw@sustc.edu.cn)

${ }^{\#}$ Co-First Author, * Corresponding Author everyone to live longer and healthy life. BSN is one of the vital ingredients with collective set of tiny and powerconstrained sensor nodes for continuous diagnosis of the patient's health to get proper and accurate medical record [1]. In BSNs, wearable devices on the one-hand playing very significant role in collecting and monitoring the patient's vitalsign signals on regular-basis with close-connection to base station (BS) [3], but on the other-hand these are facing the biggest hurdle of limited power supply and less computational capabilities [4][5]. Most of time it is hard to frequently recharge/replace the batteries of the in/on-body and digestive implantable sensors, therefore, it is one of the critical problems to reduce power utilization and extend the lifetime of BSNs. A significant contribution of information communication technologies have changed the scope of energy constrained, battery-dependent and several un-limited charging sources, but due to random nature of the external power sources, there will be no longer and continuous supply for effective monitoring of the patient's health. Even sensing activities will not be predicted with that spontaneously generated amount of power, in that situation BSNs cannot be promising except getting the higher delay and discontinuous transmission. Hence, restricted energy budget analysis with high interruption in deployment process will be highlighted [1]. At present many researchers debated a lot about the energy and battery charge consumption issues and less computational capabilities of the traditional schemes in BSN [7][6]. However, very few propose/design the energy and battery-efficient techniques for healthcare through transmission power control, energy harvesting, duty-cycle optimization, etc in BSNs, so still there is large room vacant to be filled. Generally, energy in the BSNs is harvested by several techniques e.g., environmental resources, human body, and wireless signals [1], which are inter-linked with various other factors such as, weather condition and internal working mechanism of hardware, and large amount of delay [8][9].

Wireless energy harvesting is considered as a one of the most important ingredients for provide more energy to the pervasive healthcare platforms because of availability of radio signals at everywhere [10]. However, aforementioned conventional techniques are no more convincing to reduce both the energy and battery drains during sensing, and transmission tasks. As transmission and sensing are considered as the energy and battery hungry parts of the communication, and it is very hard to forget about the major portion (i.e., 50\%) of power consumption by transmission part. In the mean-time traditional networks are not up-to-mark with high capabilities to effectively recover the battery charge during effective diagnosis, so it is very vital 
to bring state-of-the art and innovative technologies in the healthcare field [15].

Also several challenges are faced by wireless power transfer based energy harvesting schemes, hence, it is time to develop the adaptive transmission power control (TPC) enabled methods, which fairly adapt the resources with high reliability. Besides, TPC based methods not only reduce the power drain but also enhance the operational performance of the entire system. Novelty of our research lies in the integration of both TPC and duty-cycle for adaptation of power levels and execution period of tasks, respectively, for energy-efficiency and battery lifetime extension in smart healthcare system. By adopting our proposed approach the transmission power and duty-cycle will be adjusted during the node activities and dynamic wireless channel conditions with reasonable reliability (i.e., packet loss ratio) in BSNs.

This paper contributes in four distinct ways. First, a novel framework of a joint TPC and duty-cycle adaptation approach for energy-efficiency in smart healthcare system is developed. Second, adaptive energy-efficient transmission power control (AETPC) algorithm is proposed to adjust temporal variations in wireless channel during static (i.e., standing and walking at a constant speed) and dynamic (i.e., running) body postures. Third, a Feedback Control-based duty-cycle algorithm is proposed for regulating the execution period of tasks (i.e., sensing and transmission). Fourth, system-level battery and energy harvesting models are proposed for body sensor nodes by analyzing the energy consumption during sensing and transmission tasks.

The paper is further organized as follows. Section II elaborates extensive literature review. Wireless channel model is designed in Section III. Joint TPC and duty-cycle adaptation approach is developed in Section IV with several sub-sections. In Section V, experimental setup is presented. Section VI presents the conclusions.

\section{RELATED WORK}

Several researchers have already worked on TPC and WPT in BSNs. Authors in [1-3], propose the framework for medical healthcare by adopting WET and WIT techniques but did not consider the TPC, battery model and extra power budget analysis. [2-4], propose a novel energy-efficient TPC based algorithm by adapting the time-varying conditions of the wireless channel, besides they compare their proposed algorithm with the other conventional methods and prove that their research is worth applicable for the health applications. In [5], the role of 5G-enabled technologies in wearable devices is examined, but joint duty-cycle and TPC strategy is not the center of attention. Researchers in [6], present the resourceconstrained medical internet of things (M-IoT) for remote regions and predict the consequences of less supplant energy on the future healthcare facilities. Authors in [5], develop the novel green and friendly algorithms for the media streaming in the WBSN, they proved that their proposed strategies are the

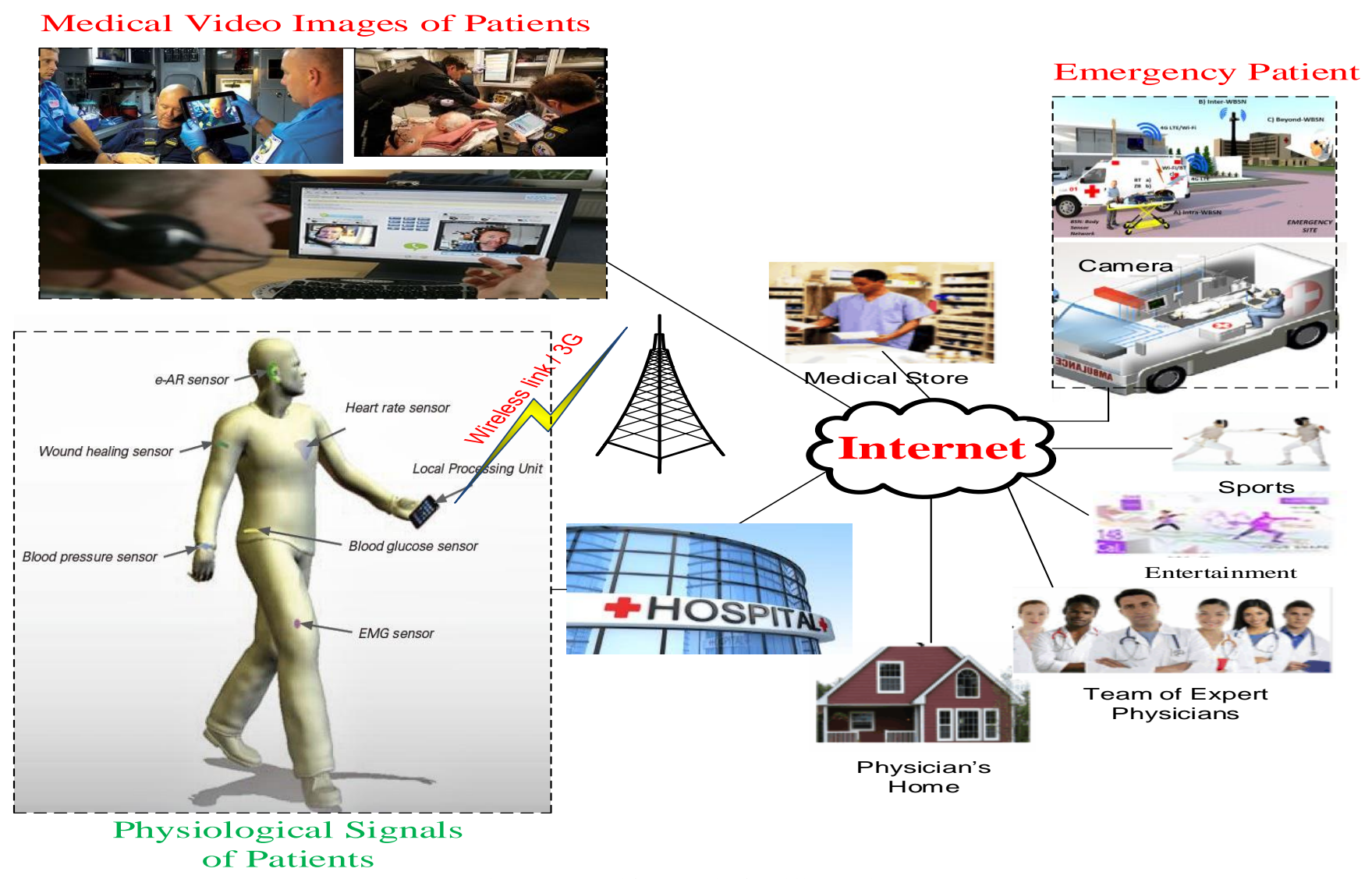

Fig. 1. Proposed framework of Mobile Healthcare 
appropriate for the health applications, but do not consider the joint TPC and duty-cycle based mechanism with the features of wireless channel.

Researchers in [6][7], design the cloud-based health monitoring and management techniques, but do not emphasis joint TPC and duty-cycle based mechanism for medical health. Besides they, discuss cardiac relevant issues in 5G-based internet of medical things, but do not taken into account the energy and power optimization problems. [8], present the role, importance and challenges of the Green medical IoT, but neglected to integrate TPC and duty-cycle in BSN. Researchers in [9], design the integrated IoT and BSN system, but totally forget to discuss the role of the power control and duty-cycle based mechanism for green and battery-efficient smart healthcare system. [10], develop the novel energy optimization model for the WBSNs, but they do not discuss the integrated TPC and duty-cycle methods with adaptive wireless channel model. In [11], design the transmission power control based energy saving algorithm over the 5G networks for the medical healthcare applications, besides they present the emerging role of $5 \mathrm{G}$ platform in minimizing the size of the sensor nodes.

Authors [12], examine the joint routing and TPC based protocols in WSN, but they do not focus at the energy saving and battery charge optimization with adaptive wireless link for BSNs. In [13], propose power control enabled high-efficiency load balancing for class-A power amplifier to support WPT system, but state-of-the art mechanism of energy conservation in healthcare is not taken into account. Researchers in [14], develop a joint duty-cycle and global TP management for energy harvesting in industrial WSNs, but oversimplified to consider the system level battery model, innovative architecture and techniques for BSN. Authors in [15][17] develop and discuss various energy optimization techniques with frameworks of Telemedicine, and battery-friendly BAN, but do not combine the TPC and duty-cycle based techniques for the smart medical healthcare. [16] design a joint TPC and relay strategy for the BANs and their proper correlation. In [18-19] develop combined user allocation and TPC approach for energy saving in femto-cell networks. [20-21], design TPC based technique for energy optimization in WSNs.

\section{WIRELESS CHANNEL}

The Received Signal Strength Indicator (RSSI) is the prominent channel performance measurement entity for sensor nodes in several applications especially healthcare. Transceiver radio is the computing and storage entity of RSSI data packets over main eight data packets related to body movement of humans [1][2]. RSSI explains the strength of received power at the $\mathrm{BS}$ and is analyzed by the time and transmission power etc. Besides, RSSI tells about the channel condition and hence the reliability by considering both the static and dynamic postures. Data packets are transferred after each interval of $200 \mathrm{~ms}$ and TP levels between $0 \mathrm{dBm}$ and $25 \mathrm{dBm}$. RSSI of $-100 \mathrm{dBm}$ is predefined to present the packet lost state. Furthermore, path loss at both static (i.e., standing and walking at constant speed) and dynamic (i.e., running) postures is computed frequency of $2.4 \mathrm{GHz}$. We examined that relatively less path loss is obtained at static and low frequency conditions than the path loss at dynamic posture and high frequency [2]. For posture data collection accelerometer is used (i.e., standing and walking at constant speed) and dynamic (i.e., running) body postures at constant and variable speed, respectively, as represented by case 1 and case 2 in Fig. 3 with better accuracy at low sampling rates. According to the law of attraction every object in the universe has a gravitational force toward the center of the earth.

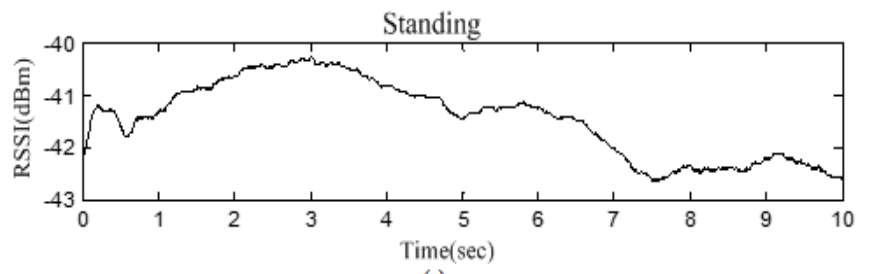

(a)

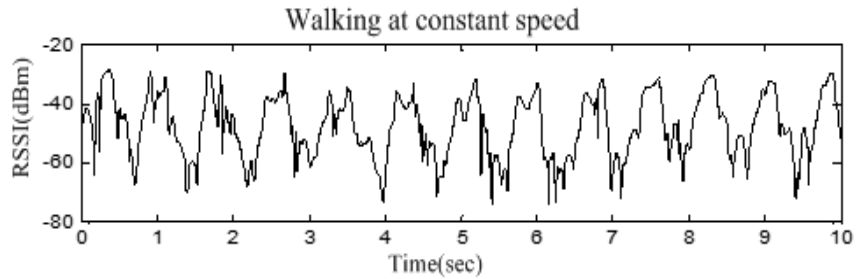

(b)

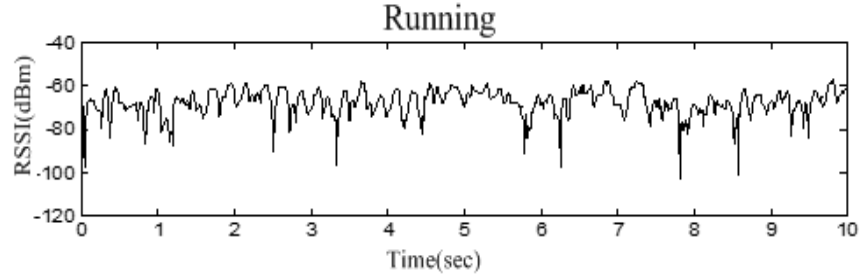

(c)

Fig. 2. RSSI measurement in different body postures, (a) \& (b) static, and (c) dynamic

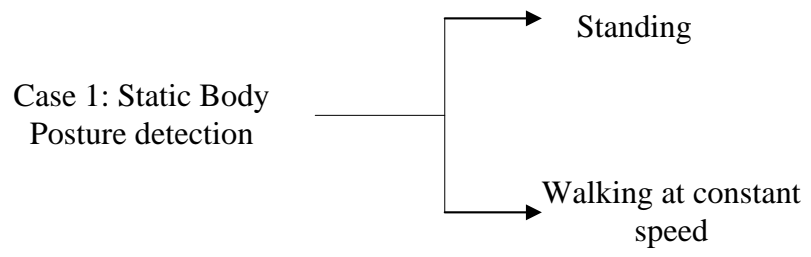

Case 2: Dynamic Body Posture detection

Fig.3. Body posture classification

Thus, we propose the TPC enabled framework by adopting static and dynamic body postures as revealed in Fig 4. The BS gets information from transmitter node than computes the RSSI according to the RSSI threshold value. When computed RSSI is greater than RSSI threshold then ACK of short interframe space period (pSIFS) delivered to transmitter without counting signaling overhead. 


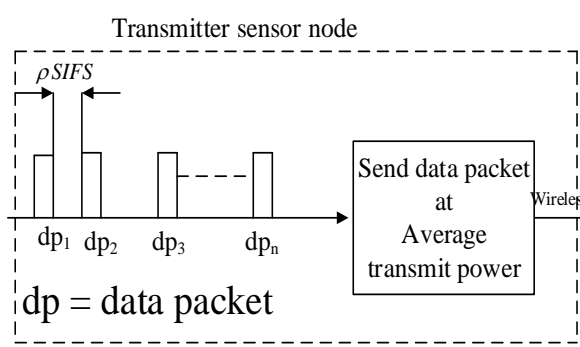

Fig. 4. TPC Framework for static Body posture detection

\section{JOINT TRANSMISSION POWER AND DUTY-CYCLE ADAPTION IN SMART HEALTHCARE}

A joint adaptive transmission power control and duty-cycle adaptation based system model for saving energy in the BSNs is proposed, where access point (AP) is considered as the key source of energy harvesting with transmitter sensor node (i.e., AP, battery, transmission power controller, and duty-cycle adapter) and base station (BS).

\section{A. System Model}

Fig.5 reveals the proposed system model with harvest-store-usecontrol (in the presence of TPC and duty-cycle adaptor) mechanism, and the BSN is configured as a star topology linked directly to the BS. In addition, energy and frequency of $1.9 \mathrm{~nJ} / \mathrm{b}$, and $2.4 \mathrm{GHz}$ in various standards such as, Bluetooth low energy (BLE) and IEEE 802.15.6,etc are adopted accordingly [18], while the modeled sensor and the reader are use $30 \mathrm{~mW}$ analog signal processor integrated circuit (IC) to manage and monitor biomedical signals [19]. Besides, energy is harvested with (AP) at harvesting rate of $\beta(t)$ by following wireless energy transfer (WET) mechanism and stored in the battery then transferred to the BS in order to save the energy. It is important to note that the instantaneous value of relying mechanism heavily impacts the harvesting amount of energy from the AP, and varies with the application type and the hardware characteristics. Therefore, can be moderately controlled as per the hardware constraints
Base station(BS)

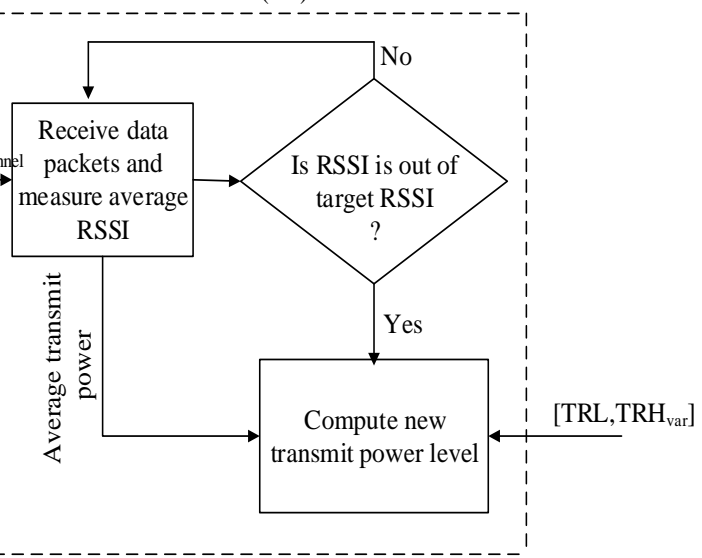

such as transmission power.

\section{B. System Level Battery Model}

The proposed model includes two types of periodic tasks namely, sensing and transmission. The period of every activity is represented as a summation of node's active time $\left(T_{O N}\right)$. The active period includes several tasks concerned to processing, sensing and operating at transceiver. The inactive time puts the system into sleep mode to save power. The energy requirement for every operation during active mode depends on the category and availability of the resources. We subordinate a value of charge $(Q)$ measured in Ampere-hour for each configuration. Similarly, the energy requirement of the transmission task relies on the output of the power amplifier (PA). The typical current $\alpha_{i}$ required by the task over a wake-up period $T_{O N}$, is given in (1).

$$
\alpha_{i}=\frac{Q_{i}}{T_{O N}}
$$

As validated in (1), the average current requirement of a task might be regulated either by adjusting the task load $\left(Q_{i}\right)$ or its execution time $\left(T_{O N}\right)$. Meanwhile, transceiver states are altered in each node including sleep, active, and idle. Henceforth, duty-cycle (DC) of sensor $S$ is specified in (2).

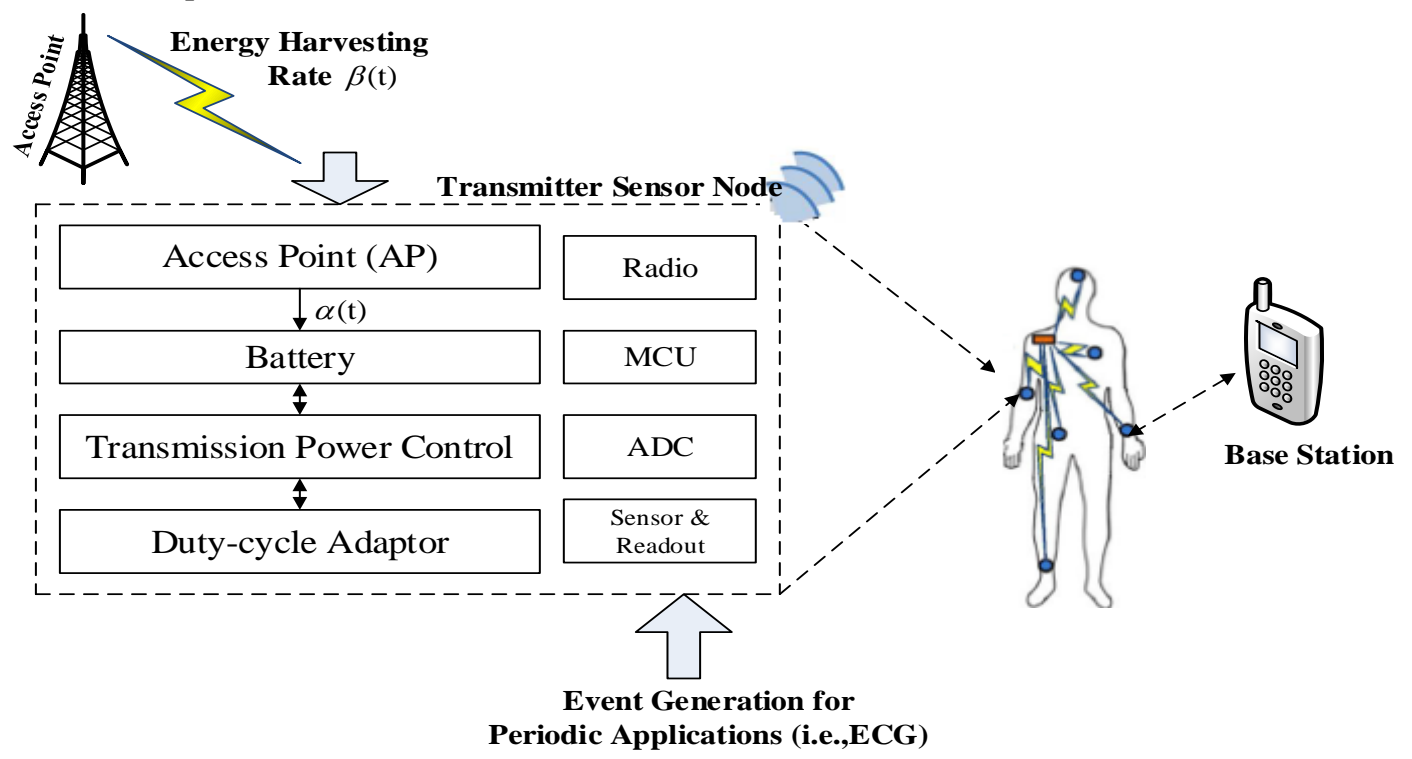

Fig. 5. Proposed Joint TPC and duty-cycle based System model for Smart Healthcare 


$$
D C_{S}=\frac{T_{O N}}{T_{O N}+T_{O F F}}
$$

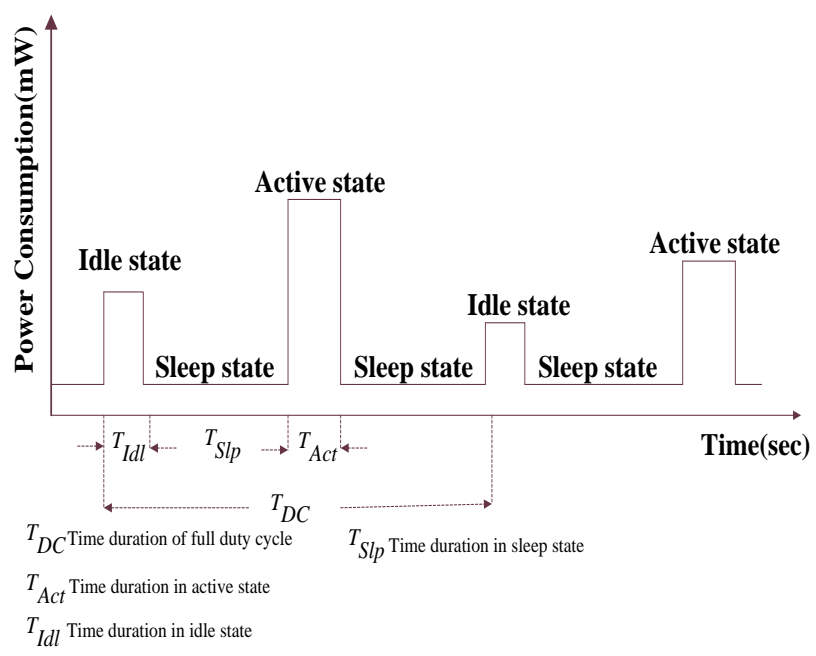

Fig. 6.Power Requirement of RF Transceiver during the Different States

Whereby

$T_{O N}:$ Active or wake up time duration of any node

$T_{\text {OFF }}$ : sleep time duration of any node

Furthermore, energy optimization is focused in healthcare applications.

For example BSN consists $S$ sensor nodes and BS with a wireless connection between them. Data can be collected, and recorded with the help of wearable devices. Due to tiny and resource-constrained nature of sensor devices it is very vital to focus at the energy drain issue in healthcare. Sensng and transmisison are the two key energy dissipation from sensor nodes $S$. A sensor ' $i$ ' where $i=1,2, \ldots \ldots, S$, depletes sensing $E_{\text {sen }}(b)$ and transmitting energy $E_{t x_{i}}\left(b, d_{i j}\right)$ during transmission of $b$ bits of data packets through $j$ sensor at distance $d_{i j}$. Cumulative energy drain is shown in eq. (3), which further guides to measure the battery state of charge (SoC). Key parameters for SoC computation are, current consumption during sensing task ( $\left.\alpha_{\text {sense }}\right)$, current consumption during transmission task $\left(\alpha_{T x}\right)$ and energy harvesting rate $(\beta)$ with future active time period $\left(T_{O N}\right)$ as in (4).

$$
E=\sum_{i=1}^{S}\left(E_{s e n_{i}}(b)+E_{t x_{i}}\left(b, d_{i j}\right)\right)
$$

$$
\operatorname{SoC}\left(D C_{S}\right)=\sum_{i=1}^{S}\left[\beta-\left(E+C_{\text {leakage }}\right)\right] \times n T_{O N}
$$

\section{Adaptive Energy-Efficient Transmission Power Control Algorithm}

We propose an adaptive energy-efficient transmission power control (AETPC) algorithm which adapts power according to time-varying wireless channel and feedback/ acknowledgment from BS. Proposed algorithm is the modified version of adaptive power control algorithm in [2], but power allocation strategy is different in both. The major components of AETPC are, lowest RSSI sample ( $\left.R_{\text {lowest }}\right)$, RSSI average $(\bar{R})$, RSSI target ( $R_{t} \arg e t$ ), averaging weight $\alpha_{1}$ of good channel, aggregate weight $\alpha_{2}$ of poor quality channel, and lower threshold value $T R L$.

Fig.7 exhibits a connection between transmission power and feedback controller (FC) and both the inputs/outputs of the distinct portions. Since, AETPC is interconnected with the FC through system state register; that gathers data about energy depletion. In addition AETPC utilizes an adaptive and ondemand mechanism to determine and accomplish the best transmission power level and constraint of the user respectively.

$$
\begin{aligned}
& \bar{R}=R_{\text {lowest }}+\left(1-\alpha_{1}\right) \times \bar{R} \\
& \bar{R}=R_{\text {lowest }}+\left(1-\alpha_{2}\right) \times \bar{R}
\end{aligned}
$$

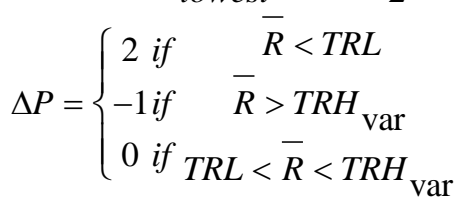

$$
\begin{aligned}
& T R H_{\mathrm{var}}=T R L+\sigma
\end{aligned}
$$

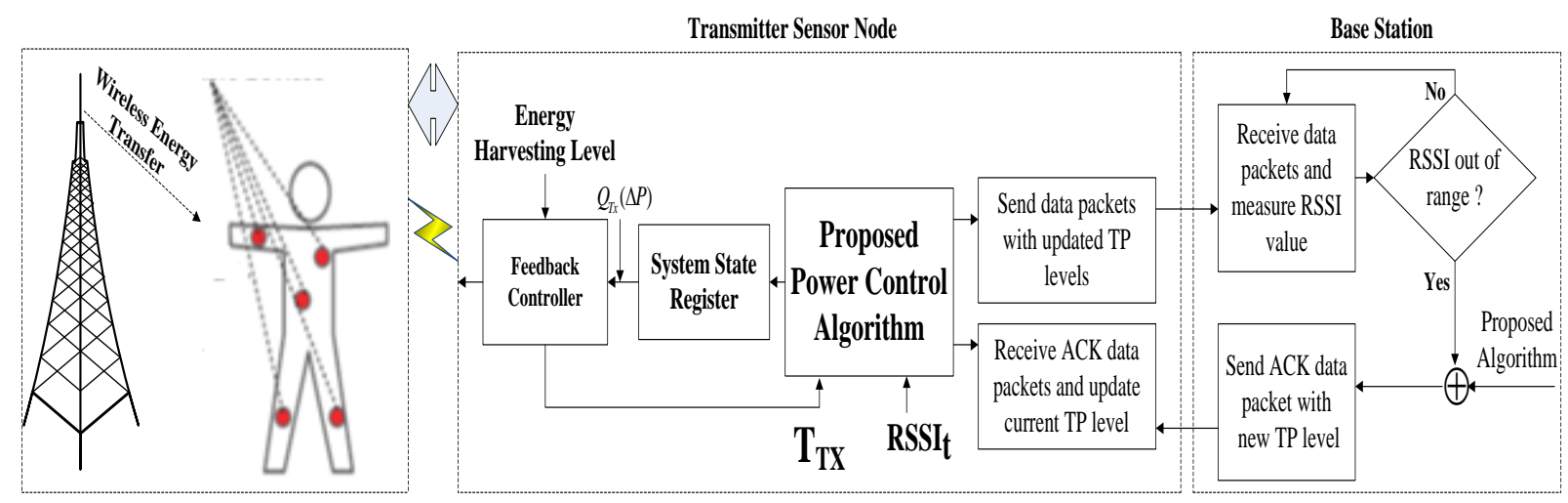

Fig. 7. Proposed Framework of Joint TPC and Feedback Controller in Smart Healthcare 


$$
\sigma=\sqrt{\frac{1}{n} \sum_{i=1}^{n}\left(R_{i}-\bar{R}\right)} \quad, \quad i=1,2, \ldots \ldots \ldots, n
$$

Whereas, $\sigma$ is the deviation (in $\mathrm{dBm}$ ) of $n$ RSSI samples as in (9), $R$ depicts RSSI samples, $\bar{R}$ reveals RSSI average, $T R L$ is lower threshold. It is assumed that $T R L=-88 \mathrm{dBm}$, $R_{t}$ arg $e t=-85 \mathrm{dBm}$, and $T R H_{\text {var }}=-83 \mathrm{dBm}$. The TP adjustment is also significant to guarantee maximum data delivery.

The foremost reason to adopt $R_{\text {lowest }}$ is to endure the communication on the basis of feedback (i.e., ACK) from BS, to compensate the sudden break-down in transmission at the loss/drop of latest RSSI samples as shown in Fig.8. Typical conventional constant TPC and PTPC methods adopt RSSI threshold values with static attributes without adopting wireless channel's features. Assume that AETPC is regulated by the BS and the transmitter node with uplink transmission of data. Transmission power and the equivalent RSSI for the lowest RSSI sample are $P_{t}, R_{\text {lowest }}=R_{\text {latest }}-1$, respectively. Subsequently, reception of the RSSI sample, the BS amends the $\bar{R}$, according to the (1) and (2) by analyzing the channel states accordingly. The developed AETPC is simple and efficient to set up with less memory and computational complexity.

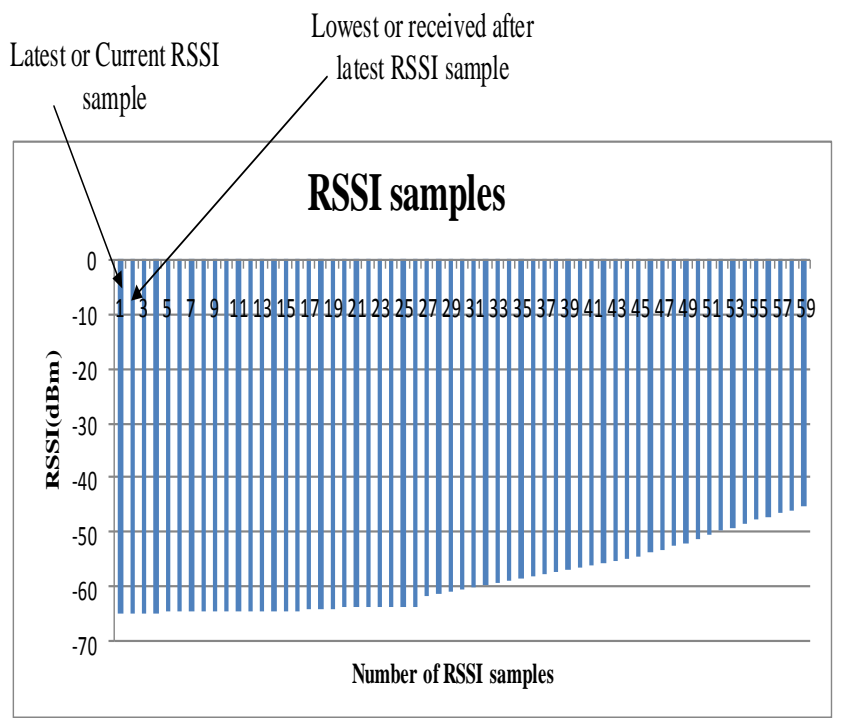

Fig. 8. Illustration of lowest and latest RSSI samples

\section{Feedback Control-Based Duty-Cycle Adaption Algorithm}

Feedback control-based duty-cycle adaptation mechanism supervises the inception rate. A node tracks a sleep-wake up cycle of the transceiver, whereas the major portion of that cycle is disbursed in the low-power sleep mode. This approach has been applied in [14] to harvest energy for industrial sensor networks but medical health is not their focus, and they only develop the heuristics approach to maintain the energy neutral condition (reduce the duty-cycle when the energy harvesting is less, and increase the duty-cycle in the presence of high scavenged energy). These methods emphasis to improve performance of nodes but do not ponder execution period of sensing and transmission tasks for entire system. A joint TPC and duty-cycle algorithm moderately integrates both ingredients, and is not applicable for rapidly varying and limited range BSNs. Duty-cycling is the most common power management method to harvest energy [14].

$$
\begin{aligned}
& \text { Inputs : } \Delta P, Q_{T x}(\Delta P), Q_{F C}, n, C_{\text {leakage }} \\
& \text { Output }: T_{T x} \\
& \text { if } \beta \geq \beta_{\text {th }} \\
& T_{T x}=\left\lceil\frac{Q_{\text {sense }}(\Delta P)+Q_{T x}(\Delta P)+Q_{F C} / n}{\beta-\left(\alpha_{\text {sense }}+\alpha_{T x}+C_{\text {leakage }}\right)}\right] \\
& \text { else } \\
& T_{T x}=\frac{Q_{T x}(\Delta P) \times D_{Z E I}}{\operatorname{SoC}(t)-\left(C_{\text {leakage }}-S o C_{\text {min }}(t)+\sigma\right) \times D_{Z E I}} \\
& \text { endif } \\
& T_{F C}=n \times T_{T x} \\
& \text { wait }\left(T_{F C}\right) \\
& \text { end }
\end{aligned}
$$

Fig.9. Feedback control-based Duty-cycle adaptation algorithm

It can be applied to the fact that embedded schemes regularly support sleeping modes, or constructive static duty-cycling mechanism for balancing both generated and utilized energies. The main goal of the proposed feedback control-based dutycycle adaptation mechanism is to compute execution duration of activities according to energy neutral operation (ENO), with persistent battery charge over time. We consider sensing and transmission tasks, and develop a FC-based duty-cycle approach. Suppose, $Q_{T x}(\Delta P)$ be the charge needed by the transmission activity when the power allocation and CPU's extra energy dissipation by the feedback controller are represented as to $\Delta P$ and $Q_{F C}$, respectively. The process for regulating the duty-cycle is represented in Fig. 9, it can be observed that a measurement of the energy by harvesting method from AP to nodes is applied initially. If the scavenged energy is greater than a definite threshold ( $\left.\beta \geq \beta_{t h}\right)$, then FC calculates the next active time period of the transmitting activity by utilizing eq.(4) and Fig.9. In that situation if the harvested energy is not sufficient to tolerate ENO, then FC will shift to negative-energy mode. In this mode $(\beta=0)$, the FC attempts to calculate the active time period of the very small activity without completely discharging the battery. In addition, FC's main responsibility is to adopt SoC of the battery $(\operatorname{SoC}(t))$ as well as an optimization of a zero-energy interval time duration shown by $D_{Z E I}$. An additional entity $\sigma$ is a deviation factor to satisfy the targeted threshold and minimize the loopholes during state of charge maximization. 


\section{EXPERIMENTAL RESULTS AND DISCUSSIONS}

In this section, we set-up the experimental platform for smart healthcare by considering wireless energy transfer (WET) concept in BSN and energy saving method with the power control and duty-cycle adaptation algorithms in MATLAB. BSN with four sensors, dynamic wireless channel, WET stage, total time $T$ of one second and the single slot $t_{0}$ of $200 \mathrm{~ms}$ are assumed [18]. Aggregated power loss value is $10^{-3}$, while supply power of the access point $P_{b}$ is $100 \mathrm{~W}$. It is assumed that our Feedback Controller is so intelligent that sensor's information (i.e., less/more energy level, battery charge level, etc) will be managed instead of specifically considering Wireless Information Transfer (WIT) step. In this process more power can be consumed in transferring acknowledgment (ACK) to access point (AP). For better and fair performance equal circuit energy drain is considered. Moreover, the realtime datasets of static (i.e., standing and walking at constant speed) and dynamic (i.e., running) body postures are used from large database NICTA [18].

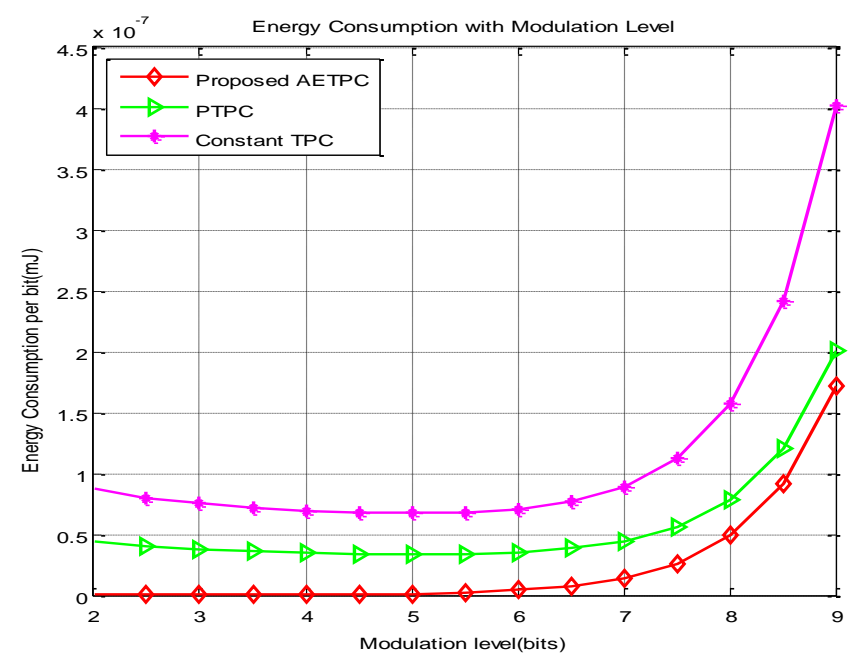

(a)

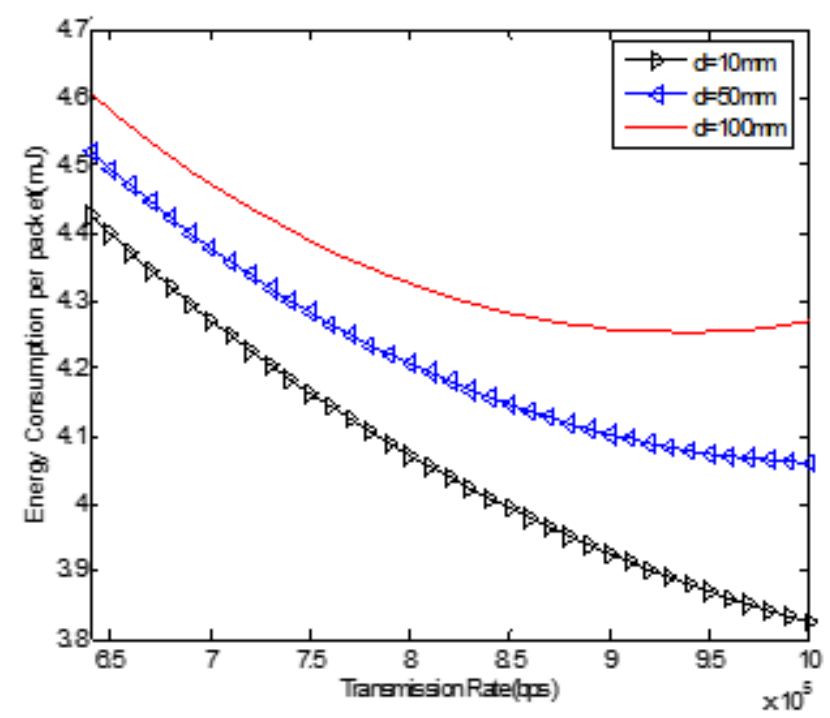

(b)

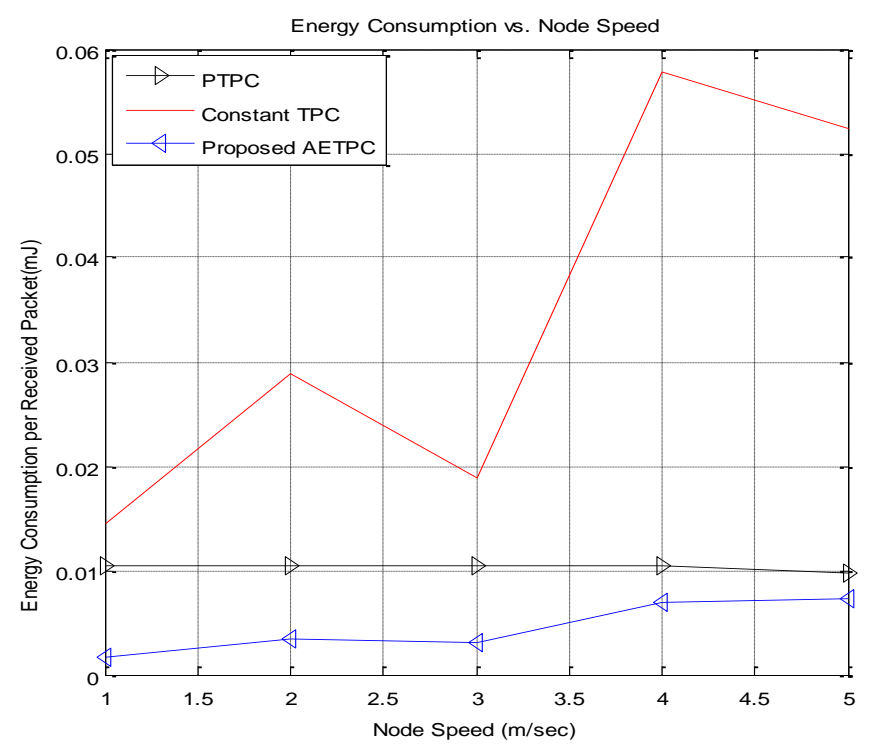

(c)

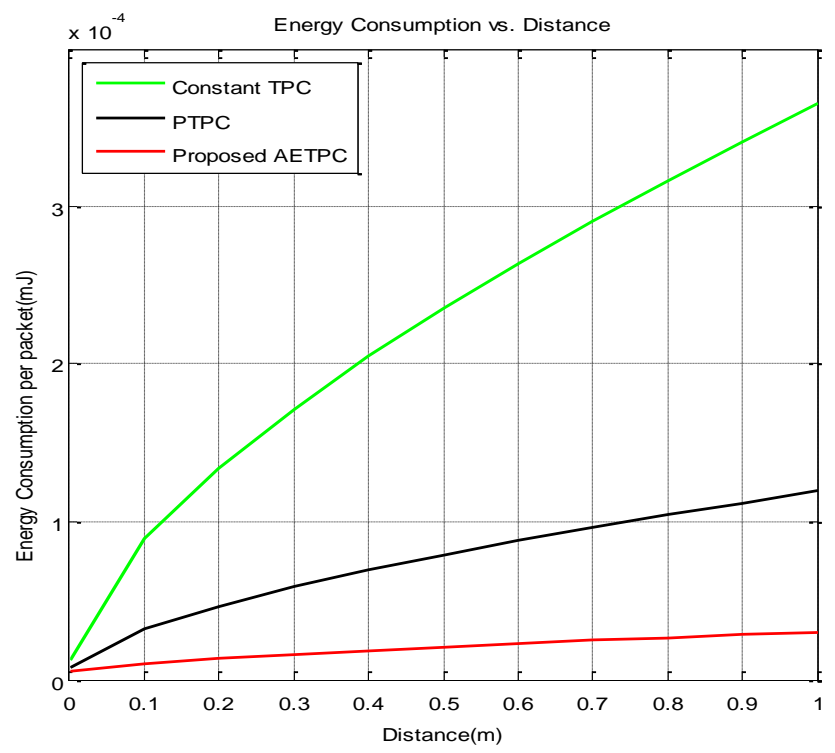

(d)

Fig.10. Relationship between energy consumption per packet/bit and, a) modulation level, b) transmission rate, c) node speed, d) distance

An old age patient with restricted movement standing, walking and running at speed of $0.15 \mathrm{~km} / \mathrm{h}, 0.5 \mathrm{~km} / \mathrm{h}$ and $2.5 \mathrm{~km} / \mathrm{h}$ respectively is involved. Fig.10 (a) shows the relationship between modulation level (in bits) and energy consumption for PTPC algorithm, Constant TPC and proposed AETPC in smart healthcare system. It is examined that energy depletion increases with the large modulation level more in constant TPC, and PTPC algorithms and less in proposed AETPC. Fig. 10 (b) shows the relationship between transmission rate and energy consumption for different distance values e.g., $10 \mathrm{~mm}$, $50 \mathrm{~mm}$ and $100 \mathrm{~mm}$. It is analyzed and revealed that by increasing distance more energy drain is obtained in an exponentially increasing fashion with respect to the data rate.

Fig.10 (c) exhibits the relationship between distance and the energy depletion per data packet for constant TPC, PTPC and proposed AETPC, in which it is examined that with the 
increase of distance energy consumption increases at higher level in constant TPC and PTPC, while relatively less in the proposed AETPC. Besides, distance of $1 \mathrm{~m}$ is adopted due the maximum range for BSNs about 5-10 m, while here in our case because of dynamic nature of wireless channel small distance is used. Furthermore, if we look at the threshold distance $d_{t h}$ for the condition $d>d_{t h}$, then total energy transmission will be main contributor of energy depletion with monotonically increasing function of R for PTPC algorithm, Constant TPC and proposed AETPC. Fig. 10 (d). We observed that as node speed increases then energy depletion gets higher for constant TPC, slightly high in the PTPC, and small in the proposed AETPC algorithms respectively

\section{CONCLUSION AND FUTURE WORK}

This research contributes in four distinct ways. First, innovative framework of a joint transmission power control (TPC) and duty-cycle adaptation method for healthcare is developed. Second, AETPC algorithm is proposed for adjusting temporal deviations in on-body wireless channel during static and dynamic body postures. Third, Feedback Control-based duty-cycle algorithm for regulating the execution period of tasks is proposed. Fourth, system-level battery and energy harvesting models for sensor nodes by analyzing the energy utilization of sensing/transmission tasks is proposed. It has also been examined and interpreted that there are marginally more deviations in dynamic postures than static ones owing to noteworthy effects of fewer body motions in later. Additionally, the developed AETPC reveals more steady RSSI level in both static and dynamic body postures than traditional constant TPC and PTPC techniques. In addition, AETPC saves more energy of $11.5 \%$ with realistic PLR. The joint TPC and FC-based duty-cycle approach satisfies the RSSI and PLR requirement in building suitable medical healthcare system. In near future, we are looking forward to develop adaptive modulation scheme particularly in cross-layer approach for healthcare systems.

\section{ACKNOWLEDGEMENT}

This work is partially supported by Natural Science Foundation of China 6171101169, National Key R\&D Plan Key Special Plan on Public Security Risk Mitigation/Response 2017YFC0804003, Technologies and Equipment Guangdong Education Bureau Fund 2017KTSCX166, the Science and Technology Innovation Committee Foundation of Shenzhen JCYJ20170817112037041, Science and Technology Innovation Committee Foundation of Shenzhen (Grant No. ZDSYS201703031748284). Also for this research Andrei Gurtov was supported by the Center for Industrial Information Technology (CENIIT) project 17.01. This work is supported in part by the HEC Pakistan under the START-UP RESEARCH GRANT PROGRAM (SRGP)\#211465/SRGP/R\&D/HEC/2016, and Sukkur IBA University, Sukkur, Sindh, Pakistan.

\section{CONFLICTS OF INTEREST}

The authors declare no conflict of interest.

\section{REFERENCES}

[1] T.Ruan, Z. Jun Chew, Energy-Aware Approaches for Energy Harvesting Powered Wireless Sensor Nodes, IEEE Sensor Journal, Vol.17, No.7, pp.2165-2173,2017

[2] X.Xiao, S.Pirbhulal, K.Dong, W.Wu, X. Mei. "Performance evaluation of plain weave and honeycomb weave electrodes for human ECG monitoring“, Journal of Sensors, Vol.17, no.1, pp.1-14, 2017

[3] S. Pirbhulal, H. Zhang, M. E. E Alahi, H. Ghayvat, S. C. Mukhopadhyay, Y.-T. Zhang, et al., "A novel secure IoT-based smart home automation system using a wireless sensor network," Sensors, Vol. 17 , pp. 69, 2016.

[4] Sodhro A.H, A.K Sangaiah, S.Pirphulal, Aicha Sekhari, Yacine Ouzrout, Green Media-Aware Medical IoT System, Multimedia Tools \& pplications,Springer,http://link.springer.com/article/10.1007/s11042018-5634-0,pp.1-20, 2018

[5] S. Pirbhulal, H. Zhang, S. C. Mukhopadhyay,Wang Wu, "An efficient biometric-based algorithm using heart rate variability for securing body sensor networks," Sensors, vol. 15, no. 7, pp. 15067-15089, 2015.

[6] E.Gonzalez , P.R, Vargas-Rosales C, Avila A, de Cerio DP , "Survey of WBSNs for Pre-Hospital Assistance: Trends to maximize the networks lifetime and video transmission techniques", MDPI Sensors, Vol.15, No.5, pp.11993-12021, 2015.

[7] A.H Sodhro, M.A Shah, "Role of 5G in Medical Health", IEEE International Conference on Innovations in Electrical Engineering and Computational Technologies (ICIEECT),Indus University, Karachi, Pakistan, 5-7 April, 2017.

[8] S. Pirbhulal, H. Zhang, M. E. E Alahi, H. Ghayvat, S. C. Mukhopadhyay, Y.-T. Zhang, et al., "A novel secure IoT-based smart home automation system using a wireless sensor network," Sensors, Vol. 17, p. 69, 2016.

[9] A.H Sodhro, S.Pirbhulal, G.H.Sodhro, "Convergence of IoT and Product Lifecycle Management in Medical Health Care "Future Generation Computer Systems, Elsevier, Vol.86 No.(2018),pp. 380-391,2018

[10] L.Wang, Ni An, A.H Sodhro,Y.Li,'Power-aware Wireless Communication System Design for BANs",E-Health Telecommunication Systems and Networks Journal, Vol. 2 No. 2, pp. 23-28, June 2013.

[11] R.Gravina, G.Fortino,"Multi-sensor fusion in body sensor networks: State-of-the-art and research challenges. Information Fusion, Vol.35, No.1, pp.68-80, 2017.

[12] S.Galzarano, G.Fortnio, "A Task-Oriented Framework for Networked Wearable Computing", IEEE Transaction on Automation Science and Engineering Vol. 13, No. 2, pp.621-638, 2016.

[13] C. Andrea, A.P T.Nhan Le, Michel Auguin, "A joint duty-cycle and transmission power management for energy harvesting WSN." IEEE Transactions on Industrial Informatics, Vol. 10, No. 2, pp. 928-936, 2014.

[14] D.Yanjie, Md.J Hossain, J.Cheng. "Joint power control and time switching for SWIPT systems with heterogeneous QoS requirements." IEEE Communications Letters, Vol.20, No. 2, pp.328$331,2016$.

[15] A.H Sodhro, A.Kumar, S.Pirbhulal, G.H Sodhro, "An Energy-Efficient Algorithm for Wearable Electrocardiogram Signal Processing in Ubiquitous Healthcare Applications", MDPI Sensors Vol.8, No.3, pp.923, 2018.

[16] E.Ibarra, A.Antonopoulos, E.Kartsakli, Joel J.P.C. Rodrigues, Christos Verikoukis, "QoS-aware Energy Management in Body Sensor Nodes Powered by Human Energy Harvesting, IEEE Sensors Journal, Vol.16, No.2, pp.542-549, 2016.

[17] A.H Sodhro, S.Pirbhulal, G.Hassan, S.Lohano Power Control Algorithms for Media Transmission in Remote Healthcare Systems, IEEE Access, Vol.6, July, 2018

[18] Y.Hao, L.Peng, H.Lu, M.M.Hassan, A.Alamri, "Energy Harvesting Based Body Area Networks for Smart Health", MDPI Sensors, Vol.17, No.7, pp.1-10, 2017.

[19] A.H sodhro, A.Kumar, G.H Sodhro, '5G-based Transmission Power Control Mechanism in Fog Computing for IoT Devices', MDPI Sustainability,Vol.10, No.4, pp.1-17, April 2018.

[20] W.Wu, S.Pirbhulal, et al, "Assessment of Biofeedback Training for Emotion Management Through Wearable Textile Physiological Monitoring System", IEEE Sensors Journal, Vol.15 No.12, pp. 70877095, 2015 